\title{
Excluding chaos by regularizing a macro model with Hicksian nonlinearity
}

\section{Xiaosheng Zhang ${ }^{1 *}$ and Maoren Yin ${ }^{2}$}

"Correspondence:

zhangxs@cnu.edu.cn

'School of Mathematical Sciences, Capital Normal University, Beijing, 100048, China

Full list of author information is available at the end of the article

\begin{abstract}
A piecewise linear macro model with ceilings and floors is perturbed in the way of regularization to interpret economic reality more reasonably. Hopf bifurcations for these perturbed models are obtained, excluding chaos which, as is well known, takes place in the unperturbed version. The study reveals the non-chaotic nature of the model.
\end{abstract}

Keywords: dynamics; bifurcations; cycles; chaos

\section{Introduction}

In this paper, we consider a macro model introduced by Simonovits (1982) [1], based on an earlier version by Honkapohja and Ito (1980) [2]. The model describes an economy with two markets, one for labor and one for consumer goods and its qualitative aspects have been analyzed by Hommes and Nusse [3] and Hommes [4].

For clarity, we briefly recall the assumptions in [1]. Let $L_{t}$ stand for the employment and $I_{t}$ the inventory at time $t$, where $t$ is a nonnegative integer. Assume that $Y_{t}^{D}=a+b L_{t}$ is the demand for goods and $Y_{t}=\delta L_{t}$ is the production, where $a, b$, and $\delta$ are positive constants. The firms are assumed to have static expectations of the demand for goods, that is, $E\left[Y_{t+1}^{D}\right]=Y_{t}^{D}$. The desired inventory $I_{t+1}^{*}$ is considered to be proportional to the expected demand, so that $I_{t+1}^{*}=\beta E\left[Y_{t+1}^{D}\right], \beta>0$. The supply and demand balance reads

$$
\left\{\begin{array}{l}
Y_{t+1}=E\left(Y_{t+1}^{D}\right)+I_{t+1}^{*}-I_{t}, \\
I_{t+1}=Y_{t+1}+I_{t}-Y_{t+1}^{D}
\end{array}\right.
$$

which implies the following linear macro model:

$$
\left\{\begin{array}{l}
L_{t+1}=\frac{1}{\delta}\left[(\beta+1)\left(a+b L_{t}\right)-I_{t}\right], \\
I_{t+1}=\frac{\delta-b}{\delta}\left[(\beta+1)\left(a+b L_{t}\right)-I_{t}\right]+I_{t}-a .
\end{array}\right.
$$

In addition, it is assumed that the productivity of labor is larger than the marginal propensity to consume, that is,

$$
\delta>b .
$$

With the above assumptions, (1.1) has the so-called Keynesian unemployment equilibrium

$$
\left(L_{t}, I_{t}\right) \equiv E_{0}(\beta):=\left(\frac{a}{\delta-b}, \frac{\beta a \delta}{\delta-b}\right)
$$

@ 2015 Zhang and Yin; licensee Springer. This is an Open Access article distributed under the terms of the Creative Commons Attribution License (http://creativecommons.org/licenses/by/4.0), which permits unrestricted use, distribution, and reproduction in any medium, provided the original work is properly credited. 
at which (1.1) has two complex eigenvalues with their product being $b(\beta+1) / \delta$. Thus when $\beta>\beta_{0} \equiv \delta / b-1, E_{0}(\beta)$ is unstable.

Now assume that the supply of labor is a constant $d$, being the full-employment ceiling and that the production at full employment $\left(L_{t}=d\right)$ is larger than demand, that is,

$$
(\delta-b) d-a>0 .
$$

Considering the constraints $0 \leq L_{t} \leq d$ and $I_{t} \geq 0$, Simonovits [1] introduced the following piecewise linear model:

$$
\left(\mathcal{M}_{0}\right)\left\{\begin{array}{l}
L_{t+1}=\min \left\{d, \max \left\{0, \frac{1}{\delta}\left[(\beta+1)\left(a+b L_{t}\right)-I_{t}\right]\right\}\right\}, \\
I_{t+1}=\max \left\{0,(\delta-b) \min \left\{d, \max \left\{0, \frac{1}{\delta}\left[(\beta+1)\left(a+b L_{t}\right)-I_{t}\right]\right\}\right\}+I_{t}-a\right\} .
\end{array}\right.
$$

This nonlinear model still has $E_{0}(\beta)$ as its unique equilibrium. By assumptions (1.2) and (1.3), it is easy to show that $E_{0}(\beta)$ has a neighborhood lying in an area where the equations of $\left(\mathcal{M}_{0}\right)$ are identical to those of (1.1) and then it is unstable for $\beta>\beta_{0}$.

With 'ceilings' and 'floors' imposed on a linear system, the nonlinearity of the model $\left(\mathcal{M}_{0}\right)$ is called a Hicksian nonlinearity. Hommes and Nusse [3] and Hommes [4] have shown that when $\beta>\beta_{0}$ there exists a stable periodic orbit, a quasi-periodic attractor or a chaotic attractor for a certain range of the parameters. The research on the dynamics of economic models with Hicksian nonlinearity has attracted many economists and mathematicians (see [5-9]).

On the other hand, it is noted that such a model as $\left(\mathcal{M}_{0}\right)$ presents some peculiarities. For example, its attractor contains a segment where $L_{t}$ equals $d$, implying the occurrence of full employment (see [4]). However, full employment is thought to be unrealistic since, ascribed to the diversity of financial restrictions, the nonexistence of a common ceiling for the production of consumption and investment goods, and so on, the non-homogeneity of employments is ubiquitous in the production sectors (see [9-12]).

Another peculiarity that needs to be mentioned is the presence of the area of pure linearity around the equilibrium, which makes the bifurcation problem mathematically degenerate and prevents one from investigating the dynamic nature of such a model (see [12]).

Thus, some methods of modifying the models with Hicksian nonlinearities have been proposed to interpret the 'ceilings' and the 'floors' more reasonably (see [10-12]). One kind of these methods is to regularize such a model by smoothing out the corners at the ceilings and the floors without substantially modifying the assumptions of the model, but including some realistic situations. The regularization method was introduced by Saura et al. (1998) [12]. In [12], a piecewise linear economic model is perturbed in the way of regularization and its non-chaotic dynamic property is proved to be persistent against smoothing, which shows that this model is non-chaotic in nature. How about the nature of the dynamics of the economy stated above? Do the chaotic behaviors of the model $\left(\mathcal{M}_{0}\right)$ survive smoothing?

Following the ideal of Saura et al. [12], in this paper the model $\left(\mathcal{M}_{0}\right)$ is regularized. Hopf bifurcations for the regularized models are obtained, excluding chaos which takes place in $\left(\mathcal{M}_{0}\right)$. The result implies that the model has a non-chaotic nature.

This paper is organized as follows. The regularized models of $\left(\mathcal{M}_{0}\right)$ and the bifurcation results are presented in the following section. The proof of the results is given in Section 3, and a bifurcation theorem used here is given in the Appendix. 
Throughout this paper, we always assume that the positive numbers $a, b, d, \delta$, and $\beta$ satisfy (1.2) and (1.3).

\section{Regularized models and bifurcations}

In this section, we first introduce the regularized models and then state the bifurcation results.

\subsection{Regularized models}

For brevity, denote $M(x)=\max \{x, 0\}, m(x)=\min \{x, d\}, \mu(x)=m(M(x))$ for $x \in \mathbb{R}$ and $\gamma(\beta, x, y)=[(\beta+1)(a+b x)-y] / \delta$ for $(x, y) \in \mathbb{R}^{2}$. Then $\left(\mathcal{M}_{0}\right)$ is rewritten into the form

$$
\left(\mathcal{M}_{0}\right) \quad\left\{\begin{array}{l}
L_{t+1}=\mu\left(\gamma\left(\beta, L_{t}, I_{t}\right)\right), \\
I_{t+1}=M\left((\delta-b) \mu\left(\gamma\left(\beta, L_{t}, I_{t}\right)\right)+I_{t}-a\right) .
\end{array}\right.
$$

Denote $w_{0}=a /(\delta-b)$ and $z_{0}=a \delta / b$, that is, $\left(w_{0}, z_{0}\right)=E_{0}\left(\beta_{0}\right)$. Then $z_{0}>0$ and, by (1.3), $w_{0} \in(0, d)$, which implies that $\left(w_{0}, z_{0}\right)$ possesses a neighborhood where, for $\beta$ close to $\beta_{0}$, the equations of $\left(\mathcal{M}_{0}\right)$ are identical to those of (1.1). This is essential for the regularization and the analysis to work.

In the light of the regularization method introduced in [12], two families of $C^{4}$-smooth functions $M_{\epsilon}(\cdot)$ and $m_{\epsilon}(\cdot)$ are employed to approximate $M(\cdot)$ and $m(\cdot)$, respectively, where $\epsilon$ is a small positive number. We assume the following hypotheses:

$\left(\mathrm{H}_{1}\right) M_{\epsilon}(x) \rightarrow M_{0}(x):=M(x)\left(\epsilon \rightarrow 0^{+}\right)$, and for $j=0,1,2,3,4$ the function $(\epsilon, x) \mapsto M_{\epsilon}^{(j)}(x)$ are continuous in a neighborhood of $(\epsilon, x)=\left(0, w_{0}\right)$ as well as in a neighborhood of $(\epsilon, x)=\left(0, z_{0}\right)$

$\left(\mathrm{H}_{2}\right) m_{\epsilon}(x) \rightarrow m_{0}(x):=m(x)\left(\epsilon \rightarrow 0^{+}\right)$, and for $j=0,1,2,3,4$ the functions $(\epsilon, x) \mapsto m_{\epsilon}^{(j)}(x)$ are continuous in a neighborhood of $(\epsilon, x)=\left(0, w_{0}\right)$;

$\left(\mathrm{H}_{3}\right) \quad 0<m_{\epsilon}^{\prime}(x), M_{\epsilon}^{\prime}(x)<1, \lim _{x \rightarrow-\infty} m_{\epsilon}^{\prime}(x)=\lim _{x \rightarrow+\infty} M_{\epsilon}^{\prime}(x)=1$;

$\left(\mathrm{H}_{4}\right)$ there exists $\underline{z}, 0<\underline{z}<\min \left\{w_{0}, z_{0}\right\}$, such that $M_{\epsilon}^{\prime \prime}(x)>0, M_{\epsilon}^{\prime \prime \prime}(x)<0$ and $M_{\epsilon}^{(4)}(x)>0$ for every $x \in(\underline{z},+\infty)$;

$\left(\mathrm{H}_{5}\right)$ there exists $\bar{w}, w_{0}<\bar{w}<d$, such that $m_{\epsilon}^{(j)}(x)<0, x \in(-\infty, \bar{w}), j=2,3,4$.

Here, the hypotheses $\left(\mathrm{H}_{1}\right)-\left(\mathrm{H}_{3}\right)$ describe the closeness between $M_{\epsilon}(\cdot)$ and $M_{0}(\cdot)$ as well as between $m_{\epsilon}(\cdot)$ and $m_{0}(\cdot)$ (see Figure 1). To explain the hypotheses $\left(\mathrm{H}_{4}\right)$ and $\left(\mathrm{H}_{5}\right)$, we notice that the step functions $M_{0}^{\prime}(x)$ and $m_{0}^{\prime}(x)$ are approximated by smooth $S$-shaped curves $M_{\epsilon}^{\prime}(x)$ and $m_{\epsilon}^{\prime}(x)$, respectively. Thus, it is natural that $M_{\epsilon}^{\prime}(x)$, as indicated in $\left(\mathrm{H}_{4}\right)$, is increasing and concave with decreasing curvature on $(\underline{z},+\infty)$ (see Figure 2). Similarly $\left(\mathrm{H}_{5}\right)$ is also a natural hypothesis for $m_{\epsilon}(x)$.

Figure 1 Smoothing the max and min functions.

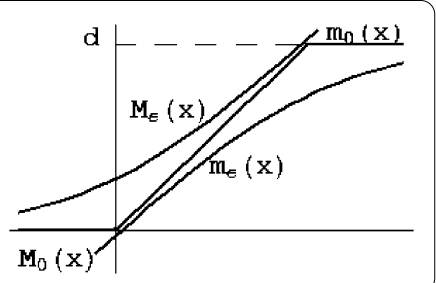




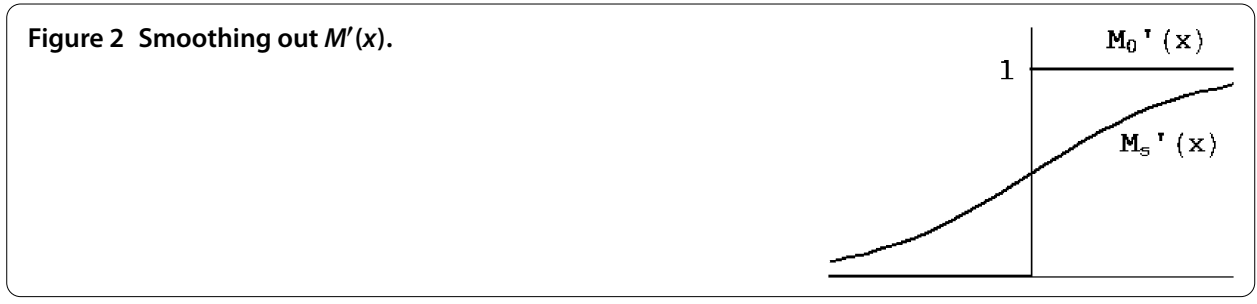

Denote $\mu_{\epsilon}(x)=m_{\epsilon}\left(M_{\epsilon}(x)\right)$ for $x \in \mathbb{R}$. By replacing $\mu(\cdot)$ and $M(\cdot)$ in $\left(\mathcal{M}_{0}\right)$ with $\mu_{\epsilon}(\cdot)$ and $M_{\epsilon}(\cdot)$, respectively, we get the following regularized model:

$$
\left(\mathcal{M}_{\epsilon}\right) \quad\left\{\begin{array}{l}
L_{t+1}=\mu_{\epsilon}\left(\gamma\left(\beta, L_{t}, I_{t}\right)\right) \\
I_{t+1}=M_{\epsilon}\left((\delta-b) \mu_{\epsilon}\left(\gamma\left(\beta, L_{t}, I_{t}\right)\right)+I_{t}-a\right) .
\end{array}\right.
$$

Here, the flexible curves $\mu_{\epsilon}(\cdot)$ and $M_{\epsilon}(\cdot)$ are employed to interpret the non-homogeneity in the labor market as well as in the consumption market. Also, it is noted that the smaller the positive number $\epsilon$ is, the closer this regularized model is to its original version $\left(\mathcal{M}_{0}\right)$.

\subsection{Bifurcations}

The first conclusion of the following theorem confirms the existence of the equilibrium for $\left(\mathcal{M}_{\epsilon}\right)$ and the second states the occurrence of the bifurcation and its stability.

Theorem 2.1 Assume that hypotheses $\left(\mathrm{H}_{1}\right)-\left(\mathrm{H}_{5}\right)$ hold. Then

(i) for $\epsilon>0$ sufficiently small and $\beta$ close to $\beta_{0},\left(\mathcal{M}_{\epsilon}\right)$ has a unique equilibrium $E_{\epsilon}(\beta)$ with $E_{\epsilon}(\beta) \rightarrow E_{0}(\beta)\left(\epsilon \rightarrow 0^{+}\right)$;

(ii) for $\epsilon>0$ sufficiently small there exist $\beta_{\epsilon}>0$ and $\eta_{\epsilon}>0$ with $\beta_{\epsilon} \rightarrow \beta_{0}\left(\epsilon \rightarrow 0^{+}\right)$such that for $\beta \in\left(\beta_{\epsilon}, \beta_{\epsilon}+\eta_{\epsilon}\right)\left(\mathcal{M}_{\epsilon}\right)$ has a locally attracting invariant closed curve surrounding the equilibrium $E_{\epsilon}(\beta)$ which shrinks to $E_{\epsilon}\left(\beta_{\epsilon}\right)$ as $\beta \rightarrow \beta_{\epsilon}$ from above. No such curve exists for $\beta \in\left(\beta_{\epsilon}-\eta_{\epsilon}, \beta_{\epsilon}\right)$.

As an illustration, we consider the following specific regularization. As in [12], let the step functions $M^{\prime}(x)$ and $m^{\prime}(x)$ be approximated by the logistic curves

$$
M_{\epsilon}^{\prime}(x)=\frac{1}{2}+\frac{1}{2} \tanh \left(\frac{x}{\epsilon}\right) \quad \text { and } \quad m_{\epsilon}^{\prime}(x)=\frac{1}{2}-\frac{1}{2} \tanh \left(\frac{x-d}{\epsilon}\right),
$$

respectively. Thus

$$
\begin{aligned}
& M_{\epsilon}(x)=\frac{\epsilon}{2} \ln \left[\exp \left(\frac{x}{\epsilon}\right)+\exp \left(\frac{-x}{\epsilon}\right)\right]+\frac{x}{2} \\
& m_{\epsilon}(x)=-\frac{\epsilon}{2} \ln \left[\exp \left(\frac{x-d}{\epsilon}\right)+\exp \left(-\frac{x-d}{\epsilon}\right)\right]+\frac{x+d}{2} .
\end{aligned}
$$

It is easy to check that $\left(\mathrm{H}_{1}\right)-\left(\mathrm{H}_{5}\right)$ are satisfied.

Set the data of the parameters by $\epsilon=0.01, a=0.095, b=0.9, d=1$, and $\delta=1$. For $\beta=$ $0.1,0.2,0.4$, and 0.45 , different orbits are plotted in Figure 3 and Figure 4. Each of the orbits contains 5,000 points after a transient time of 50. Figure 3 shows numerically that the equilibrium is stable when $\beta=0.1$ and that invariant closed curves appear when $\beta=0.2$ or $\beta=0.45$. A 37-periodic obit is observed for $\beta=0.4$ (Figure 4). 
Figure 3 Invariant curves.

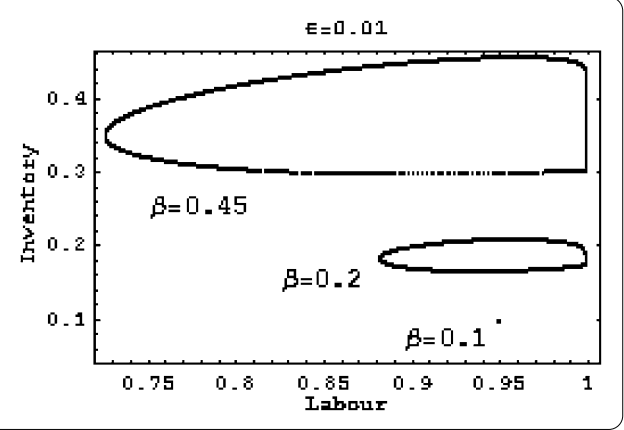

Figure 4 A periodic orbit.

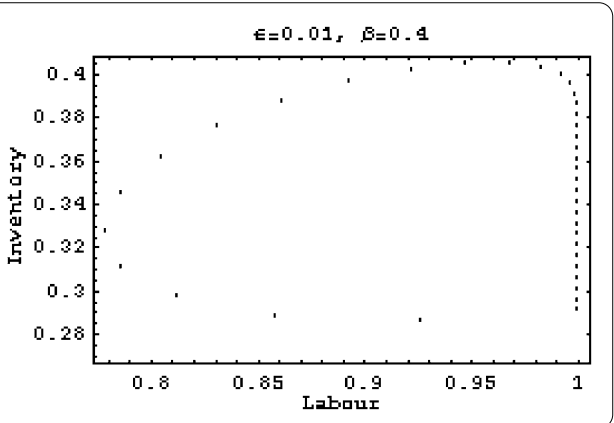

\subsection{Comments}

From the above theorem we know that for $\epsilon>0$ sufficiently small and $\beta>\beta_{\epsilon}$ near $\beta_{\epsilon}$, the asymptotic behavior of the model $\left(\mathcal{M}_{\epsilon}\right)$ is represented by the motion on the invariant closed curve, which is closely related to an important notion called the rotation number. When the rotation number is a rational number $p / q$ the system has periodic obits with period $q$. When this number is irrational, there are no periodic orbits and every orbit is dense in the closed curve, giving rise to a quasi-periodic behavior [13]. Moreover, since the rotation number depends continuously on the parameters, the dynamics inside the invariant curve shifts back and forth from periodic to quasi-periodic behavior as $\beta$ or $\epsilon$ change.

It is well known that periodic or quasi-periodic behaviors are non-chaotic, meaning that there are no periodic points with different periods, and the orbits that start close remain close for all times. The result implies that the model has a non-chaotic nature.

\section{Proof of Theorem 2.1}

In this section, it is always assumed that the hypotheses $\left(\mathrm{H}_{1}\right)-\left(\mathrm{H}_{5}\right)$ hold.

Set $\mathbb{R}^{+}=(0,+\infty), S=\mathbb{R}^{+} \times \mathbb{R}^{+}$. For simplicity, we make the change of parameters by letting

$$
\beta=\frac{k \delta}{b}-1 \quad \text { and } \quad \hat{\gamma}(k, \cdot, \cdot):=\gamma\left(\frac{k \delta}{b}-1, \cdot, \cdot\right) .
$$

Thus to prove Theorem 2.1, we turn to the bifurcation problem for the mapping $\Phi_{\epsilon}(k, \cdot, \cdot)$ : $S \mapsto S$,

$$
\Phi_{\epsilon}(k, x, y)=\left(\begin{array}{c}
\mu_{\epsilon}(\hat{\gamma}(k, x, y)) \\
M_{\epsilon}\left((\delta-b) \mu_{\epsilon}(\hat{\gamma}(k, x, y))+y-a\right)
\end{array}\right)^{T} .
$$


Next, as in [14], this system will be transformed equivalently into a special form of mapping so that the bifurcation problem can be handled easily. To do this, we consider the mapping $\pi_{\epsilon}(k, \cdot, \cdot): S \mapsto S$,

$$
\pi_{\epsilon}(k, x, y)=\left(y, M_{\epsilon}\left((\delta-b) \mu_{\epsilon}(\hat{\gamma}(k, x, y))+y-a\right)\right) .
$$

Let $W$ denote the image of $S$ under the mapping $\pi_{\epsilon}(k, \cdot, \cdot)$. From $\left(\mathrm{H}_{3}\right)$, we see that both of $\mu_{\epsilon}(\cdot)$ and $M_{\epsilon}(\cdot)$ are invertible. Then $\pi_{\epsilon}(k, \cdot, \cdot)$ is a homeomorphism with its inverse mapping $\pi_{\epsilon}^{-1}(k, \cdot, \cdot): W \mapsto S$,

$$
\pi_{\epsilon}^{-1}(k, u, v)=\left(\frac{1}{k}\left[\mu_{\epsilon}^{-1}\left(\frac{M_{\epsilon}^{-1}(v)-u+a}{\delta-b}\right)+\frac{u}{\delta}-\frac{a k}{b}\right], u\right) .
$$

By calculating, it can be checked that

$$
\pi_{\epsilon} \circ \Phi_{\epsilon} \circ \pi_{\epsilon}^{-1}(u, v)=F_{\epsilon}(k, u, v):=\left(v, G_{\epsilon}(k, u, v)\right), \quad(u, v) \in W
$$

where $G_{\epsilon}(k, \cdot, \cdot)$ is defined by

$$
\begin{aligned}
& G_{\epsilon}(k, u, v)=M_{\epsilon}\left(\psi_{\epsilon}(k, u, v)\right), \\
& \psi_{\epsilon}(k, u, v)=(\delta-b) \mu_{\epsilon}\left(\varphi_{\epsilon}(k, u, v)\right)+v-a, \\
& \varphi_{\epsilon}(k, u, v)=\frac{k}{\delta-b}\left(M_{\epsilon}^{-1}(v)-u+a\right)-\frac{v}{\delta}+\frac{a k}{b} .
\end{aligned}
$$

Then the mappings $\Phi_{\epsilon}(k, \cdot, \cdot): S \mapsto S$ and $F_{\epsilon}(k, \cdot, \cdot): W \mapsto W$ are conjugate by $\pi_{\epsilon}(k, \cdot, \cdot)$ : $S \mapsto W$, and then we only need to study the bifurcation of the mapping $F_{\epsilon}(k, \cdot, \cdot)$.

Noticing that $\varphi_{0}\left(1, z_{0}, z_{0}\right)=w_{0}$ and $\psi_{0}\left(1, z_{0}, z_{0}\right)=z_{0}$, by $\left(\mathrm{H}_{1}\right)$ and $\left(\mathrm{H}_{2}\right)$ we get the following lemma.

Lemma 3.1 The functions $\varphi_{\epsilon}(\cdot, \cdot, \cdot), \psi_{\epsilon}(\cdot, \cdot, \cdot)$ and $G_{\epsilon}(\cdot, \cdot, \cdot)$ have continuous fourth-order partial derivatives in a neighborhood of $(\epsilon, k, u, v)=\left(0,1, z_{0}, z_{0}\right)$.

For $s$ in a neighborhood of $s=z_{0}$, consider the equation

$$
\xi(\epsilon, k, s) \equiv s-G_{\epsilon}(k, s, s)=0 \text {. }
$$

Noticing that

$$
\xi\left(0,1, z_{0}\right)=0, \quad \xi_{s}\left(0,1, z_{0}\right)=1-\frac{b}{\delta}>0
$$

and applying the implicit function theorem, we find that for $(\epsilon, k)$ in a neighborhood of $(\epsilon, k)=(0,1)$, (3.4) has a unique zero, denoted by $z_{\epsilon, k}$, in a neighborhood of $s=z_{0}$. Moreover, $z_{\epsilon, k}$ is continuous jointly in $(\epsilon, k)$ and is continuously differentiable with respect to $k$. Obviously, $\left(z_{\epsilon, k}, z_{\epsilon, k}\right)$ is a fixed point of the mapping $F_{\epsilon}(k, u, v)$. That is, the following lemma holds. 
Lemma 3.2 For $(\epsilon, k)$ in a neighborhood of $(\epsilon, k)=(0,1), F_{\epsilon}(k, \cdot, \cdot)$ has unique fixed point $\left(z_{\epsilon, k}, z_{\epsilon, k}\right) \in W$. Moreover, $z_{\epsilon, k}$ is continuous jointly in $(\epsilon, k)$ and is continuously differentiable with respect to $k$.

Remark 3.1 Set $E_{\epsilon}(\beta)=\pi_{\epsilon}^{-1}\left(k, z_{\epsilon, k}, z_{\epsilon, k}\right)$ with $\beta=k \delta / b-1$. Then for $\epsilon>0$ sufficiently small and $\beta$ close to $\beta_{0},\left(\mathcal{M}_{\epsilon}\right)$ has a unique equilibrium $E_{\epsilon}(\beta)$. Moreover, it is easy to check that $z_{0, k}=a \delta(k \delta-b)[b(\delta-b)]^{-1}$ and that $\pi_{0}^{-1}\left(k, z_{0, k}, z_{0, k}\right)=E_{0}(\beta)$. Since $z_{\epsilon, k} \rightarrow z_{0, k}\left(\epsilon \rightarrow 0^{+}\right)$, we have correspondingly $E_{\epsilon}(\beta) \rightarrow E_{0}(\beta)\left(\epsilon \rightarrow 0^{+}\right)$. Thus the conclusion (i) of Theorem 2.1 holds.

The following lemma confirms the occurrence of the bifurcation at the equilibrium.

Lemma 3.3 For $\epsilon>0$ sufficiently small there exist $k_{\epsilon}$ and $\eta_{\epsilon}^{\prime}$ with $k_{\epsilon} \rightarrow 1\left(\epsilon \rightarrow 0^{+}\right)$such that for every $k \in\left(k_{\epsilon}-\eta_{\epsilon}^{\prime}, k_{\epsilon}\right)$ or $k \in\left(k_{\epsilon}, k_{\epsilon}+\eta_{\epsilon}^{\prime}\right)$, the mapping $F_{\epsilon}(k, \cdot, \cdot)$ has an invariant closed curve surrounding the equilibrium point $\left(z_{\epsilon, k}, z_{\epsilon, k}\right)$.

Proof For $(u, v) \in W$, by calculating, we have

$$
\begin{aligned}
\frac{\partial}{\partial u} G_{\epsilon}(k, u, v)= & -k M_{\epsilon}^{\prime}\left(\psi_{\epsilon}(k, u, v)\right) \mu_{\epsilon}^{\prime}\left(\varphi_{\epsilon}(k, u, v)\right), \\
\frac{\partial}{\partial v} G_{\epsilon}(k, u, v)= & M_{\epsilon}^{\prime}\left(\psi_{\epsilon}(k, u, v)\right) \\
& \times\left\{(\delta-b) \mu_{\epsilon}^{\prime}\left(\varphi_{\epsilon}(k, u, v)\right)\left[\frac{k}{(\delta-b) M_{\epsilon}^{\prime}(v)}-\frac{1}{\delta}\right]+1\right\} .
\end{aligned}
$$

Noticing that $z_{0, k}>0$, by Lemma 3.1 we have

$$
\left\{\begin{array}{l}
\varphi_{\epsilon}\left(k, z_{\epsilon, k}, z_{\epsilon, k}\right) \rightarrow \varphi_{0}\left(k, z_{0, k}, z_{0, k}\right) \equiv w_{0}, \\
\psi_{\epsilon}\left(k, z_{\epsilon, k}, z_{\epsilon, k}\right) \rightarrow \psi_{0}\left(k, z_{0, k}, z_{0, k}\right) \equiv z_{0, k}
\end{array} \quad\left(\epsilon \rightarrow 0^{+}\right),\right.
$$

which, by $\left(\mathrm{H}_{1}\right)$, leads to

$$
\lim _{\epsilon \rightarrow 0^{+}} M_{\epsilon}^{\prime}\left(z_{\epsilon, k}\right)=\lim _{\epsilon \rightarrow 0^{+}} M_{\epsilon}^{\prime}\left(\psi_{\epsilon}\left(k, z_{\epsilon, k}, z_{\epsilon, k}\right)\right)=\lim _{\epsilon \rightarrow 0^{+}} \mu_{\epsilon}^{\prime}\left(\varphi_{\epsilon}\left(k, z_{\epsilon, k}, z_{\epsilon, k}\right)\right)=1 .
$$

It follows from (3.5) that

$$
\left.\frac{\partial G_{\epsilon}(k, u, v)}{\partial u}\right|_{\left(k, z_{\epsilon, k}, z_{\epsilon, k}\right)} \rightarrow-k,\left.\quad \frac{\partial G_{\epsilon}(k, u, v)}{\partial v}\right|_{\left(k, z_{\epsilon, k}, z_{\epsilon, k}\right)} \rightarrow k+\frac{b}{\delta} \quad\left(\epsilon \rightarrow 0^{+}\right) .
$$

Let $D F_{\epsilon}\left(k, z_{\epsilon, k}, z_{\epsilon, k}\right)$ denote the tangent mapping of $F_{\epsilon}(k, \cdot, \cdot)$ at $(u, v)=\left(z_{\epsilon, k}, z_{\epsilon, k}\right)$, then

$$
D F_{\epsilon}\left(k, z_{\epsilon, k}, z_{\epsilon, k}\right) \rightarrow T:=\left(\begin{array}{cc}
0 & 1 \\
-k & k+\frac{b}{\delta}
\end{array}\right) \quad\left(\epsilon \rightarrow 0^{+}\right)
$$

Noticing that

$$
\Delta:=4 k \delta^{2}-(b+k \delta)^{2} \rightarrow(\delta-b)(b+3 \delta)>0 \quad(k \rightarrow 1),
$$


we see that, for $k$ sufficiently close to $1, T$ has two conjugate complex eigenvalues $\lambda_{0}(k)$ and $\bar{\lambda}_{0}(k)$ with

$$
\lambda_{0}(k) \equiv \frac{b+k \delta+i \sqrt{\Delta}}{2 \delta} .
$$

Moreover, we have $\left|\lambda_{0}(k)\right|=k^{1 / 2}$, which implies that, for a small positive number $\eta_{1}$,

$$
\left|\lambda_{0}\left(1-\eta_{1}\right)\right|<1<\left|\lambda_{0}\left(1+\eta_{1}\right)\right|
$$

By Lemmas 3.1 and 3.2, the eigenvalues of $D F_{\epsilon}\left(k, z_{\epsilon, k}, z_{\epsilon, k}\right)$ depend continuously on $(\epsilon, k)$, which means that one of the eigenvalues, denoted by $\lambda_{\epsilon}(k)$, tends to $\lambda_{0}(k)$ as $\epsilon \rightarrow 0^{+}$. In view of (3.9), we see that, for $\epsilon>0$ sufficiently small,

$$
\left|\lambda_{\epsilon}\left(1-\eta_{1}\right)\right|<1<\left|\lambda_{\epsilon}\left(1+\eta_{1}\right)\right|
$$

It follows that there exists $k_{\epsilon} \in\left(1-\eta_{1}, 1+\eta_{1}\right)$ such that $\left|\lambda_{\epsilon}\left(k_{\epsilon}\right)\right|=1$.

We claim that $k_{\epsilon} \rightarrow 1$ as $\epsilon \rightarrow 0^{+}$. Otherwise there exist $\epsilon_{n}, n=1,2, \ldots$, with $\epsilon_{n} \rightarrow 0^{+}$ $(n \rightarrow \infty)$, such that $k_{\epsilon_{n}} \rightarrow \hat{k} \neq 1(n \rightarrow \infty)$, which implies $1=\left|\lambda_{\epsilon_{n}}\left(k_{\epsilon_{n}}\right)\right| \rightarrow\left|\lambda_{0}(\hat{k})\right|=\hat{k}^{1 / 2} \neq 1$ as $n \rightarrow \infty$, a contradiction.

Thus $\lambda_{\epsilon}\left(k_{\epsilon}\right) \rightarrow \lambda_{0}(1)$ as $\epsilon \rightarrow 0^{+}$. Noticing that $\operatorname{Re}\left(\lambda_{0}(1)\right)>0, \operatorname{Im}\left(\lambda_{0}(1)\right)>0$, and $\lambda_{0}^{\prime}(1)>0$, we see from Lemma 3.2 that, for $\epsilon>0$ sufficiently small,

$$
\operatorname{Re} \lambda_{\epsilon}\left(k_{\epsilon}\right)>0, \quad \operatorname{Im} \lambda_{\epsilon}\left(k_{\epsilon}\right)>0 \text { and }\left.\frac{d\left|\lambda_{\epsilon}(k)\right|}{d k}\right|_{k=k_{\epsilon}}>0 \text {, }
$$

which, by Theorem A.1, implies that there exists $\eta_{\epsilon}^{\prime}>0$ such that for every $k \in\left(k_{\epsilon}-\eta_{\epsilon}^{\prime}, k_{\epsilon}\right)$ or $k \in\left(k_{\epsilon}, k_{\epsilon}+\eta_{\epsilon}^{\prime}\right), F_{\epsilon}(k, \cdot, \cdot)$ has an invariant closed curve surrounding the equilibrium point $\left(z_{\epsilon, k_{\epsilon}}, z_{\epsilon, k_{\epsilon}}\right)$.

Now, we determine the direction of the bifurcation and its stability. Denote

$$
\begin{array}{lrl}
z_{\epsilon}=z_{\epsilon}, k_{\epsilon}, & \tau_{\epsilon}=\varphi_{\epsilon}\left(k_{\epsilon}, z_{\epsilon}, z_{\epsilon}\right), & \sigma_{\epsilon}=\psi_{\epsilon}\left(k_{\epsilon}, z_{\epsilon}, z_{\epsilon}\right) \quad \text { and } \\
p_{\epsilon}=M_{\epsilon}^{\prime \prime \prime}\left(\sigma_{\epsilon}\right), & q_{\epsilon}=M_{\epsilon}^{\prime \prime \prime}\left(\tau_{\epsilon}\right), & r_{\epsilon}=m_{\epsilon}^{\prime \prime \prime}\left(M_{\epsilon}\left(\tau_{\epsilon}\right)\right) .
\end{array}
$$

From (3.6), we see that

$$
z_{\epsilon} \rightarrow z_{0}, \quad \sigma_{\epsilon} \rightarrow z_{0}, \quad \tau_{\epsilon} \rightarrow w_{0} \quad\left(\epsilon \rightarrow 0^{+}\right) .
$$

By $\left(\mathrm{H}_{1}\right)$ and $\left(\mathrm{H}_{2}\right)$, it follows that $p_{\epsilon}, q_{\epsilon}$, and $r_{\epsilon}$ are infinitesimal as $\epsilon \rightarrow 0^{+}$and

$$
M_{\epsilon}^{\prime}\left(z_{\epsilon}\right) \rightarrow 1, \quad M_{\epsilon}^{\prime}\left(\sigma_{\epsilon}\right) \rightarrow 1, \quad \mu_{\epsilon}^{\prime}\left(\tau_{\epsilon}\right) \rightarrow 1 \quad\left(\epsilon \rightarrow 0^{+}\right) .
$$

We have the following comparisons.

\section{Lemma 3.4}

(i) $\left[M_{\epsilon}^{\prime \prime}\left(\sigma_{\epsilon}\right)\right]^{2}=o\left(p_{\epsilon}\right)\left(\epsilon \rightarrow 0^{+}\right)$; 
(ii) $\left[M_{\epsilon}^{\prime \prime}\left(\tau_{\epsilon}\right)\right]^{2}=o\left(q_{\epsilon}\right)\left(\epsilon \rightarrow 0^{+}\right)$;

(iii) $\left[m_{\epsilon}^{\prime \prime}\left(M_{\epsilon}\left(\tau_{\epsilon}\right)\right)\right]^{2}=o\left(r_{\epsilon}\right)\left(\epsilon \rightarrow 0^{+}\right)$;

(iv) $\left[M_{\epsilon}^{\prime \prime}\left(z_{\epsilon}\right)\right]^{2}=o\left(p_{\epsilon}\right)\left(\epsilon \rightarrow 0^{+}\right)$.

Proof From (3.10) and $\left(\mathrm{H}_{4}\right)$, we can assume that $z_{\epsilon}, \sigma_{\epsilon}>\underline{z}$. By $\left(\mathrm{H}_{4}\right)$, we have $M_{\epsilon}^{\prime \prime}(x)>0$, $M_{\epsilon}^{\prime \prime \prime}(x)<0$, and $M_{\epsilon}^{(4)}(x)>0$ for $x>\sigma_{\epsilon}$. Then

$$
\begin{aligned}
0 & >\frac{1}{p_{\epsilon}}\left[M_{\epsilon}^{\prime \prime}\left(\sigma_{\epsilon}\right)\right]^{2}=-\int_{\sigma_{\epsilon}}^{\infty} 2 M_{\epsilon}^{\prime \prime}(s) \frac{M_{\epsilon}^{\prime \prime \prime}(s)}{p_{\epsilon}} d s \\
& \geq-2 \int_{\sigma_{\epsilon}}^{\infty} M_{\epsilon}^{\prime \prime}(s) d s=-2\left(1-M_{\epsilon}^{\prime}\left(\sigma_{\epsilon}\right)\right) .
\end{aligned}
$$

It follows from (3.11) that the conclusion (i) holds. In the same way, we can prove (ii) and, by using $\left(\mathrm{H}_{5}\right)$, (iii) can be derived similarly.

Lastly, in view of $\left(\mathrm{H}_{1}\right),\left(\mathrm{H}_{3}\right)$, and $\left(\mathrm{H}_{4}\right)$, it is easy to see that $M_{\epsilon}(x)>M(x)$ for $x \in \mathbb{R}$. Then $z_{\epsilon}=M_{\epsilon}\left(\sigma_{\epsilon}\right)>M_{0}\left(\sigma_{\epsilon}\right)=\sigma_{\epsilon}$ and then $0<M_{\epsilon}^{\prime \prime}\left(z_{\epsilon}\right)<M_{\epsilon}^{\prime \prime}\left(\sigma_{\epsilon}\right)$ which, by (i), implies (iv).

Further, by using the above lemma, we can make the following useful comparisons. In view of the derivative formula

$$
\mu_{\epsilon}^{\prime \prime}\left(\tau_{\epsilon}\right)=m_{\epsilon}^{\prime \prime}\left(M_{\epsilon}\left(\tau_{\epsilon}\right)\right)\left(M_{\epsilon}^{\prime}\left(\tau_{\epsilon}\right)\right)^{2}+m_{\epsilon}^{\prime}\left(M_{\epsilon}\left(\tau_{\epsilon}\right)\right) M_{\epsilon}^{\prime \prime}\left(\tau_{\epsilon}\right)
$$

and the inequality $2|p q| \leq p^{2}+q^{2}$ for $p, q \in \mathbb{R}$, and by using (ii) and (iii), we get

$$
\left[\mu_{\epsilon}^{\prime \prime}\left(\tau_{\epsilon}\right)\right]^{2}=o\left(q_{\epsilon}\right)+o\left(r_{\epsilon}\right) \quad\left(\epsilon \rightarrow 0^{+}\right)
$$

which, combining with (i) and (iv), respectively, leads to

$$
\mu_{\epsilon}^{\prime \prime}\left(\tau_{\epsilon}\right) M_{\epsilon}^{\prime \prime}\left(\sigma_{\epsilon}\right)=o\left(p_{\epsilon}\right)+o\left(q_{\epsilon}\right)+o\left(r_{\epsilon}\right) \quad\left(\epsilon \rightarrow 0^{+}\right)
$$

and

$$
\mu_{\epsilon}^{\prime \prime}\left(\tau_{\epsilon}\right) M_{\epsilon}^{\prime \prime}\left(z_{\epsilon}\right)=o\left(p_{\epsilon}\right)+o\left(q_{\epsilon}\right)+o\left(r_{\epsilon}\right) \quad\left(\epsilon \rightarrow 0^{+}\right)
$$

In addition, we have

$$
\begin{aligned}
\mu_{\epsilon}^{\prime \prime \prime}\left(\tau_{\epsilon}\right)= & m_{\epsilon}^{\prime \prime \prime}\left(M_{\epsilon}\left(\tau_{\epsilon}\right)\right)\left(M_{\epsilon}^{\prime}\left(\tau_{\epsilon}\right)\right)^{3}+m_{\epsilon}^{\prime}\left(M_{\epsilon}\left(\tau_{\epsilon}\right)\right) M_{\epsilon}^{\prime \prime \prime}\left(\tau_{\epsilon}\right) \\
& +3 m_{\epsilon}^{\prime \prime}\left(M_{\epsilon}\left(\tau_{\epsilon}\right)\right) M_{\epsilon}^{\prime \prime}\left(\tau_{\epsilon}\right) M_{\epsilon}^{\prime}\left(\tau_{\epsilon}\right)
\end{aligned}
$$

By using (ii), (iii), and (3.11), it follows that

$$
\mu_{\epsilon}^{\prime \prime \prime}\left(\tau_{\epsilon}\right)=q_{\epsilon}+r_{\epsilon}+o\left(q_{\epsilon}\right)+o\left(r_{\epsilon}\right) \quad\left(\epsilon \rightarrow 0^{+}\right)
$$

Lemma 3.5 The invariant curve derived from Lemma 3.3 is attracting and the bifurcation is supercritical. 
Proof Denote

$$
G_{i j}(\epsilon)=\left.\frac{\partial^{i+j} G_{\epsilon}\left(k_{\epsilon}, \cdot, \cdot\right)}{\partial u^{i} \partial v^{j}}\right|_{\left(z_{\epsilon}, z_{\epsilon}\right)}, \quad i, j=0,1,2,3, i+j=1,2,3 .
$$

By calculation, we have

$$
\begin{aligned}
G_{20}(\epsilon)= & k_{\epsilon}^{2} M_{\epsilon}^{\prime \prime}\left(\sigma_{\epsilon}\right)\left[\mu_{\epsilon}^{\prime}\left(\tau_{\epsilon}\right)\right]^{2}+\frac{k_{\epsilon}^{2}}{\delta-b} M_{\epsilon}^{\prime}\left(\sigma_{\epsilon}\right) \mu_{\epsilon}^{\prime \prime}\left(\tau_{\epsilon}\right), \\
G_{11}(\epsilon)= & -k_{\epsilon} M_{\epsilon}^{\prime \prime}\left(\sigma_{\epsilon}\right) \mu_{\epsilon}^{\prime}\left(\tau_{\epsilon}\right)\left\{(\delta-b) \mu_{\epsilon}^{\prime}\left(\tau_{\epsilon}\right)\left[\frac{k_{\epsilon}}{(\delta-b) M_{\epsilon}^{\prime}\left(z_{\epsilon}\right)}-\frac{1}{\delta}\right]+1\right\} \\
& -k_{\epsilon} M_{\epsilon}^{\prime}\left(\sigma_{\epsilon}\right) \mu_{\epsilon}^{\prime \prime}\left(\tau_{\epsilon}\right)\left[\frac{k_{\epsilon}}{(\delta-b) M_{\epsilon}^{\prime}\left(z_{\epsilon}\right)}-\frac{1}{\delta}\right] \\
G_{02}(\epsilon)= & M_{\epsilon}^{\prime \prime}\left(\sigma_{\epsilon}\right)\left\{(\delta-b) \mu_{\epsilon}^{\prime}\left(\tau_{\epsilon}\right)\left[\frac{k_{\epsilon}}{(\delta-b) M_{\epsilon}^{\prime}\left(z_{\epsilon}\right)}-\frac{1}{\delta}\right]+1\right\}^{2} \\
& +M_{\epsilon}^{\prime}\left(\sigma_{\epsilon}\right)\left\{(\delta-b) \mu_{\epsilon}^{\prime \prime}\left(\tau_{\epsilon}\right)\left[\frac{k_{\epsilon}}{(\delta-b) M_{\epsilon}^{\prime}\left(z_{\epsilon}\right)}-\frac{1}{\delta}\right]^{2}-k_{\epsilon} \mu_{\epsilon}^{\prime}\left(\tau_{\epsilon}\right) \frac{M_{\epsilon}^{\prime \prime}\left(z_{\epsilon}\right)}{\left[M_{\epsilon}^{\prime}\left(z_{\epsilon}\right)\right]^{2}}\right\} .
\end{aligned}
$$

It follows from (3.13)-(3.15) and (3.11) that

$$
\left(G_{i j}(\epsilon)\right)^{2}=o\left(p_{\epsilon}\right)+o\left(q_{\epsilon}\right)+o\left(r_{\epsilon}\right) \quad\left(\epsilon \rightarrow 0^{+}\right), i+j=2 .
$$

Further, by calculating,

$$
\begin{aligned}
G_{30}(\epsilon)= & -k_{\epsilon}^{3}\left\{M_{\epsilon}^{\prime \prime \prime}\left(\sigma_{\epsilon}\right)\left[\mu_{\epsilon}^{\prime}\left(\tau_{\epsilon}\right)\right]^{3}+\frac{1}{\delta-b} M_{\epsilon}^{\prime \prime}\left(\sigma_{\epsilon}\right) \mu_{\epsilon}^{\prime \prime}\left(\tau_{\epsilon}\right)\right. \\
& \left.+\frac{1}{(\delta-b)^{2}} M_{\epsilon}^{\prime \prime}\left(\sigma_{\epsilon}\right) \mu_{\epsilon}^{\prime}\left(\tau_{\epsilon}\right) \mu_{\epsilon}^{\prime \prime}\left(\tau_{\epsilon}\right)+\frac{1}{(\delta-b)^{2}} M_{\epsilon}^{\prime}\left(\sigma_{\epsilon}\right) \mu_{\epsilon}^{\prime \prime \prime}\left(\tau_{\epsilon}\right)\right\} \\
G_{21}(\epsilon)= & k_{\epsilon}^{2} M_{\epsilon}^{\prime \prime \prime}\left(\sigma_{\epsilon}\right)\left[\mu_{\epsilon}\left(\tau_{\epsilon}\right)\right]^{2}\left\{(\delta-b) \mu_{\epsilon}^{\prime}\left(\tau_{\epsilon}\right)\left[\frac{k_{\epsilon}}{(\delta-b) M_{\epsilon}^{\prime}\left(z_{\epsilon}\right)}-\frac{1}{\delta}\right]+1\right\} \\
& +2 k_{\epsilon}^{2} M_{\epsilon}^{\prime \prime}\left(\sigma_{\epsilon}\right) \mu_{\epsilon}^{\prime}\left(\tau_{\epsilon}\right) \mu_{\epsilon}^{\prime \prime}\left(\tau_{\epsilon}\right)\left[\frac{k_{\epsilon}}{(\delta-b) M_{\epsilon}^{\prime}\left(z_{\epsilon}\right)}-\frac{1}{\delta}\right] \\
& +\frac{k_{\epsilon}^{2}}{\delta-b} M_{\epsilon}^{\prime \prime}\left(\sigma_{\epsilon}\right) \mu_{\epsilon}^{\prime \prime}\left(\tau_{\epsilon}\right)\left\{(\delta-b) \mu_{\epsilon}^{\prime}\left(\tau_{\epsilon}\right)\left[\frac{k_{\epsilon}}{(\delta-b) M_{\epsilon}^{\prime}\left(z_{\epsilon}\right)}-\frac{1}{\delta}\right]+1\right\} \\
& +\frac{k_{\epsilon}^{2}}{\delta-b} M_{\epsilon}^{\prime}\left(\sigma_{\epsilon}\right) \mu_{\epsilon}^{\prime \prime \prime}\left(\tau_{\epsilon}\right)\left[\frac{1}{(\delta-b) M_{\epsilon}^{\prime}\left(z_{\epsilon}\right)}-\frac{k^{\prime}}{\delta}\right] \\
G_{12}(\epsilon)= & -k_{\epsilon} M_{\epsilon}^{\prime \prime \prime}\left(\sigma_{\epsilon}\right) \mu_{\epsilon}^{\prime}\left(\tau_{\epsilon}\right)\left\{(\delta-b) \mu_{\epsilon}^{\prime}\left(\tau_{\epsilon}\right)\left[\frac{k_{\epsilon}}{(\delta-b) M_{\epsilon}^{\prime}\left(z_{\epsilon}\right)}-\frac{1}{\delta}\right]+1\right\} \\
& -2 k_{\epsilon} M_{\epsilon}^{\prime \prime}\left(\sigma_{\epsilon}\right) \mu_{\epsilon}^{\prime \prime}\left(\tau_{\epsilon}\right)\left\{(\delta-b) \mu_{\epsilon}^{\prime}\left(\tau_{\epsilon}\right)\left[\frac{k_{\epsilon}}{(\delta-b) M_{\epsilon}^{\prime}\left(z_{\epsilon}\right)}-\frac{1}{\delta}\right]+1\right\} \\
& +k_{\epsilon} M_{\epsilon}^{\prime \prime}\left(\sigma_{\epsilon}\right) \mu_{\epsilon}^{\prime}\left(\tau_{\epsilon}\right)\left\{(\delta-b) \mu_{\epsilon}^{\prime \prime}\left(\tau_{\epsilon}\right)\left[\frac{k_{\epsilon}}{(\delta-b) M_{\epsilon}^{\prime}\left(z_{\epsilon}\right)}-\frac{1}{\delta}\right]+k_{\epsilon} \mu_{\epsilon}^{\prime}\left(\tau_{\epsilon}\right) \frac{M_{\epsilon}^{\prime \prime}\left(z_{\epsilon}\right)}{\left.\left[M_{\epsilon}^{\prime}\left(z_{\epsilon}\right)\right]^{2}\right\}}\right. \\
& {\left[\frac{k_{\epsilon}}{(\delta-b) M_{\epsilon}^{\prime}\left(z_{\epsilon}\right)}-\frac{1}{\delta}\right] }
\end{aligned}
$$




$$
\begin{aligned}
& -k_{\epsilon} M_{\epsilon}^{\prime}\left(\sigma_{\epsilon}\right)\left\{\mu_{\epsilon}^{\prime \prime \prime}\left(\tau_{\epsilon}\right)\left[\frac{k_{\epsilon}}{(\delta-b) M_{\epsilon}^{\prime}\left(z_{\epsilon}\right)}-\frac{1}{\delta}\right]^{2}-\frac{k_{\epsilon}}{\delta-b} \mu_{\epsilon}^{\prime \prime}\left(\tau_{\epsilon}\right) \frac{M_{\epsilon}^{\prime \prime}\left(z_{\epsilon}\right)}{\left[M_{\epsilon}^{\prime}\left(z_{\epsilon}\right)\right]^{2}}\right\}, \\
& G_{03}(\epsilon)=M_{\epsilon}^{\prime \prime \prime}\left(\sigma_{\epsilon}\right)\left\{(\delta-b) \mu_{\epsilon}^{\prime}\left(\tau_{\epsilon}\right)\left[\frac{k_{\epsilon}}{(\delta-b) M_{\epsilon}^{\prime}\left(z_{\epsilon}\right)}-\frac{1}{\delta}\right]+1\right\}^{3} \\
& +2 M_{\epsilon}^{\prime \prime}\left(\sigma_{\epsilon}\right)\left\{(\delta-b) \mu_{\epsilon}^{\prime}\left(\tau_{\epsilon}\right)\left[\frac{k_{\epsilon}}{(\delta-b) M_{\epsilon}^{\prime}\left(z_{\epsilon}\right)}-\frac{1}{\delta}\right]+1\right\} \\
& \times\left\{(\delta-b) \mu_{\epsilon}^{\prime \prime}\left(\tau_{\epsilon}\right)\left[\frac{k_{\epsilon}}{(\delta-b) M_{\epsilon}^{\prime}\left(z_{\epsilon}\right)}-\frac{1}{\delta}\right]^{2}-k_{\epsilon} \mu_{\epsilon}^{\prime}\left(\tau_{\epsilon}\right) \frac{M_{\epsilon}^{\prime \prime}\left(z_{\epsilon}\right)}{\left[M_{\epsilon}^{\prime}\left(z_{\epsilon}\right)\right]^{2}}\right\} \\
& +M_{\epsilon}^{\prime \prime}\left(\sigma_{\epsilon}\right)\left\{(\delta-b) \mu_{\epsilon}^{\prime}\left(\tau_{\epsilon}\right)\left[\frac{k_{\epsilon}}{(\delta-b) M_{\epsilon}^{\prime}\left(z_{\epsilon}\right)}-\frac{1}{\delta}\right]+1\right\} \\
& \times\left\{(\delta-b) \mu_{\epsilon}^{\prime \prime}\left(\tau_{\epsilon}\right)\left[\frac{k_{\epsilon}}{(\delta-b) M_{\epsilon}^{\prime}\left(z_{\epsilon}\right)}-\frac{1}{\delta}\right]^{2}-k_{\epsilon} \mu_{\epsilon}^{\prime}\left(\tau_{\epsilon}\right) \frac{M_{\epsilon}^{\prime \prime}\left(z_{\epsilon}\right)}{\left[M_{\epsilon}^{\prime}\left(z_{\epsilon}\right)\right]^{2}}\right\} \\
& +M_{\epsilon}^{\prime}\left(\sigma_{\epsilon}\right)\left\{(\delta-b) \mu_{\epsilon}^{\prime \prime \prime}\left(\tau_{\epsilon}\right)\left[\frac{k_{\epsilon}}{(\delta-b) M_{\epsilon}^{\prime}\left(z_{\epsilon}\right)}-\frac{1}{\delta}\right]^{3}\right. \\
& +2 k_{\epsilon} \mu_{\epsilon}^{\prime \prime}\left(\tau_{\epsilon}\right)\left[\frac{k_{\epsilon}}{(\delta-b) M_{\epsilon}^{\prime}\left(z_{\epsilon}\right)}-\frac{1}{\delta}\right] \frac{M_{\epsilon}^{\prime \prime}\left(z_{\epsilon}\right)}{\left[M_{\epsilon}^{\prime}\left(z_{\epsilon}\right)\right]^{2}} \\
& +k_{\epsilon} \mu_{\epsilon}^{\prime \prime}\left(\tau_{\epsilon}\right) \frac{M_{\epsilon}^{\prime \prime}\left(z_{\epsilon}\right)}{\left[M_{\epsilon}^{\prime}\left(z_{\epsilon}\right)\right]^{2}}\left[\frac{k_{\epsilon}}{(\delta-b) M_{\epsilon}^{\prime}\left(z_{\epsilon}\right)}-\frac{1}{\delta}\right] \\
& \left.+k_{\epsilon} \mu_{\epsilon}^{\prime}\left(\tau_{\epsilon}\right) \frac{-M_{\epsilon}^{\prime \prime \prime}\left(z_{\epsilon}\right)\left[M_{\epsilon}^{\prime}\left(z_{\epsilon}\right)\right]^{2}+2\left[M_{\epsilon}^{\prime \prime}\left(z_{\epsilon}\right)\right]^{2} M_{\epsilon}^{\prime}\left(z_{\epsilon}\right)}{\left[M_{\epsilon}^{\prime}\left(z_{\epsilon}\right)\right]^{4}}\right\} .
\end{aligned}
$$

Denote $n_{\epsilon}=M_{\epsilon}^{\prime \prime \prime}\left(z_{\epsilon}\right)$. It follows from Lemma 3.4 and (3.13)-(3.16) that, as $\epsilon \rightarrow 0^{+}$,

$$
\begin{aligned}
& G_{30}(\epsilon)=-p_{\epsilon}-\left(\frac{1}{\delta-b}\right)^{2} q_{\epsilon}+o\left(p_{\epsilon}\right)+o\left(q_{\epsilon}\right)+o\left(r_{\epsilon}\right), \\
& G_{03}(\epsilon)=(1+b / \delta)^{3} p_{\epsilon}+\frac{b^{3}\left(q_{\epsilon}+r_{\epsilon}\right)}{(\delta-b)^{2} \delta^{3}}-n_{\epsilon}+o\left(p_{\epsilon}\right)+o\left(q_{\epsilon}\right)+o\left(r_{\epsilon}\right), \\
& G_{21}(\epsilon)=(1+b / \delta) p_{\epsilon}+\frac{b\left(q_{\epsilon}+r_{\epsilon}\right)}{(\delta-b)^{2} \delta}+o\left(p_{\epsilon}\right)+o\left(q_{\epsilon}\right)+o\left(r_{\epsilon}\right), \\
& G_{12}(\epsilon)=-(1+b / \delta)^{2} p_{\epsilon}+\frac{b^{2}\left(q_{\epsilon}+r_{\epsilon}\right)}{(\delta-b)^{2} \delta^{2}}+o\left(p_{\epsilon}\right)+o\left(q_{\epsilon}\right)+o\left(r_{\epsilon}\right) .
\end{aligned}
$$

Let

$$
\begin{aligned}
a_{20}(\epsilon)= & \frac{1}{2}\left(G_{20}(\epsilon)+G_{02}(\epsilon) \cdot \lambda^{2}\left(k_{\epsilon}\right)+2 G_{11}(\epsilon) \cdot \lambda\left(k_{\epsilon}\right)\right) \frac{i}{\left(\lambda\left(k_{\epsilon}\right)-\bar{\lambda}\left(k_{\epsilon}\right)\right)^{2}}, \\
a_{02}(\epsilon)= & \frac{1}{2}\left(G_{20}(\epsilon)+G_{02}(\epsilon) \cdot \bar{\lambda}^{2}\left(k_{\epsilon}\right)+2 G_{11}(\epsilon) \cdot \bar{\lambda}\left(k_{\epsilon}\right)\right) \frac{i}{\left(\lambda\left(k_{\epsilon}\right)-\bar{\lambda}\left(k_{\epsilon}\right)\right)^{2}}, \\
a_{11}(\epsilon)= & \left(G_{20}(\epsilon)+G_{02}(\epsilon) \cdot \lambda\left(k_{\epsilon}\right) \bar{\lambda}\left(k_{\epsilon}\right)+G_{11}(\epsilon) \cdot\left(\lambda\left(k_{\epsilon}\right)+\bar{\lambda}\left(k_{\epsilon}\right)\right)\right) \cdot \frac{i}{\left(\lambda\left(k_{\epsilon}\right)-\bar{\lambda}\left(k_{\epsilon}\right)\right)^{2}}, \\
a_{21}(\epsilon)= & \left(G_{30}(\epsilon)+G_{03}(\epsilon) \lambda^{2}\left(k_{\epsilon}\right) \bar{\lambda}\left(k_{\epsilon}\right)+G_{12}(\epsilon) \lambda\left(k_{\epsilon}\right)\left(\lambda\left(k_{\epsilon}\right)+2 \bar{\lambda}\left(k_{\epsilon}\right)\right)\right. \\
& \left.+G_{21}(\epsilon)\left(\bar{\lambda}\left(k_{\epsilon}\right)+2 \lambda\left(k_{\epsilon}\right)\right)\right) \cdot \frac{-1 / 2}{\left(\lambda\left(k_{\epsilon}\right)-\bar{\lambda}\left(k_{\epsilon}\right)\right)^{3}},
\end{aligned}
$$




$$
\begin{aligned}
& Q(\epsilon)=\frac{\left|a_{11}(\epsilon)\right|^{2}}{1-\bar{\lambda}\left(k_{\epsilon}\right)}+\frac{2\left|a_{02}(\epsilon)\right|^{2}}{\lambda^{2}\left(k_{\epsilon}\right)-\bar{\lambda}\left(k_{\epsilon}\right)}+\frac{2 \lambda\left(k_{\epsilon}\right)-1}{\lambda\left(k_{\epsilon}\right)\left(1-\lambda\left(k_{\epsilon}\right)\right)} \cdot a_{11}(\epsilon) \cdot a_{20}(\epsilon)+a_{21}(\epsilon), \\
& c_{1}(\epsilon)=\operatorname{Re}\left(Q(\epsilon) \cdot \bar{\lambda}\left(k_{\epsilon}\right)\right) .
\end{aligned}
$$

From (3.17), we see that

$$
\left|a_{i j}(\epsilon)\right|^{2}=o\left(p_{\epsilon}\right)+o\left(q_{\epsilon}\right)+o\left(r_{\epsilon}\right) \quad\left(\epsilon \rightarrow 0^{+}\right), i+j=2 .
$$

On the other hand, by using (3.18), we obtain

$$
\operatorname{Re}\left(a_{21}(\epsilon) \cdot \bar{\lambda}\left(k_{\epsilon}\right)\right)=\frac{1}{16 k_{2}^{2}}\left[p_{\epsilon}+\frac{q_{\epsilon}+r_{\epsilon}}{\delta(\delta-b)}\right]+o\left(p_{\epsilon}\right)+o\left(q_{\epsilon}\right)+o\left(r_{\epsilon}\right) \quad\left(\epsilon \rightarrow 0^{+}\right) .
$$

From (3.19) and (3.20), we see that, for $\epsilon>0$ sufficiently small,

$$
\operatorname{sign}\left(c_{1}(\epsilon)\right)=\operatorname{sign}\left(\frac{1}{16 k_{2}^{2}}\left[p_{\epsilon}+\frac{q_{\epsilon}+r_{\epsilon}}{\delta(\delta-b)}\right]\right)<0
$$

By Theorem A.1, the conclusion of the lemma follows.

Remark 3.2 In view of (3.2), the above lemma implies the conclusion (ii) of Theorem 2.1. Thus the proof is complete.

\section{Appendix}

The following statement is a special case of Hopf's theorem for mappings, which is employed to prove Theorem 2.1.

Theorem A.1 (Hopf bifurcation theorem for plane mappings [12,14]) Let $F: R \times R^{2} \rightarrow R^{2}$ be a mapping of class $C^{4}$ of the form $F(k, x, y)=(y, G(k, x, y))$ which satisfies:

(i) For every $k \in R$, there exists $\tilde{z}(k)$ such that $F(k, \tilde{z}(k), \tilde{z}(k))=(\tilde{z}(k), \tilde{z}(k))$.

(ii) $D F(k, \tilde{z}(k), \tilde{z}(k))$ has complex eigenvalues $\lambda(k), \bar{\lambda}(k)$ such that $\left|\lambda\left(k_{0}\right)\right|=1$,

$$
\left(\lambda\left(k_{0}\right)\right)^{m} \neq 1, \quad m=1,2,3,4 \quad \text { and }\left.\quad \eta^{\prime}\left(k_{0}\right) \stackrel{\text { def }}{=} \frac{|\lambda(k)|}{d k}\right|_{k=k_{0}}>0(<0) .
$$

Then the mapping $F(k, \cdot, \cdot)$ has, for every $k<k_{0}$ or $k>k_{0}$ sufficiently close to $k_{0}$, an invariant closed curve surrounding the equilibrium point $(\tilde{z}(k), \tilde{z}(k))$.

The direction $\left(k<k_{0}\right.$ or $\left.k>k_{0}\right)$ of the bifurcation and its stability properties can be obtained as follows: Let us denote

$$
G_{i j}=\frac{\partial^{i+j} G}{\partial x^{i} \partial y^{j}}(k, \tilde{z}(k), \tilde{z}(k))
$$

and let

$$
\begin{aligned}
& a_{20}(k)=\frac{1}{2}\left(G_{20}+G_{02} \cdot \lambda^{2}(k)+2 G_{11} \cdot \lambda(k)\right) \frac{i}{(\lambda(k)-\bar{\lambda}(k))^{2}}, \\
& a_{02}(k)=\frac{1}{2}\left(G_{20}+G_{02} \cdot \bar{\lambda}^{2}(k)+2 G_{11} \cdot \bar{\lambda}(k)\right) \frac{i}{(\lambda(k)-\bar{\lambda}(k))^{2}},
\end{aligned}
$$




$$
\begin{aligned}
a_{11}(k)= & \left(G_{20}+G_{02} \cdot \lambda(k) \bar{\lambda}(k)+G_{11} \cdot(\lambda(k)+\bar{\lambda}(k))\right) \cdot \frac{i}{(\lambda(k)-\bar{\lambda}(k))^{2}}, \\
a_{21}(k)= & \left(G_{30}+G_{03} \lambda^{2}(k) \bar{\lambda}(k)+G_{12} \lambda(k)(\lambda(k)+2 \bar{\lambda}(k))\right. \\
& \left.+G_{21}(\bar{\lambda}(k)+2 \lambda(k))\right) \cdot \frac{-1 / 2}{(\lambda(k)-\bar{\lambda}(k))^{3}}, \\
Q(k)= & \frac{\left|a_{11}(k)\right|^{2}}{1-\bar{\lambda}(k)}+\frac{2\left|a_{02}(k)\right|^{2}}{\lambda^{2}(k)-\bar{\lambda}(k)}+\frac{2 \lambda(k)-1}{\lambda(k)(1-\lambda(k))} \cdot a_{11}(k) \cdot a_{20}(k)+a_{21}(k), \\
c_{1}(k)= & \operatorname{Re}(Q(k) \cdot \bar{\lambda}(k)) .
\end{aligned}
$$

Then, if $c_{1}\left(k_{0}\right)<0$ (respectively, $\left.c_{1}\left(k_{0}\right)>0\right)$, the invariant curve is attracting (respectively, repelling) and if $c_{1}\left(k_{0}\right) \cdot \eta^{\prime}\left(k_{0}\right)<0$ (respectively, $\left.c_{1}\left(k_{0}\right) \cdot \eta^{\prime}\left(k_{0}\right)>0\right)$, the bifurcation is supercritical (respectively, subcritical).

\section{Competing interests}

The authors declare that they have no competing interests.

\section{Authors' contributions}

All authors completed the paper together. All authors read and approved the final manuscript.

\section{Author details}

'School of Mathematical Sciences, Capital Normal University, Beijing, 100048, China. ${ }^{2}$ Department of Mathematics, Xinzhou Teachers University, Xinzhou, 034000, China.

\section{Acknowledgements}

The authors thank the referees for their important and valuable comments. This paper is supported by the scientific research projects of Beijing Municipal Commission of Education (KZ201310028031, KM201410028002).

Received: 27 December 2014 Accepted: 30 March 2015 Published online: 08 April 2015

\section{References}

1. Simonovits, A: Buffer stocks and naive expectations in a non-Walrasian dynamic macro-model: stability, cyclicity and chaos. Scand. J. Econ. 84, 571-581 (1982)

2. Honkapohja, S, Ito, T: Inventory dynamics in a simple macroeconomic model. Scand. J. Econ. 82, 184-198 (1980)

3. Hommes, $\mathrm{CH}$, Nusse, HE: Does an unstable Keynesian unemployment equilibrium in a non-Walrasian dynamic macroeconomic model imply chaos? Scand. J. Econ. 91, 161-167 (1989)

4. Hommes, CH: Chaotic Dynamics in Economic Models: Some Simple Cases Studies. Groningen Theses in Economics, Management and Organization. Wolters-Noordhoff, Groningen (1991)

5. Hicks, JR: A Contribution to the Theory of the Trade Cycle. Clarendon, Oxford (1950)

6. Gallegati, M, Gardini, L, Puu, T, Sushko, I: Hicks' trade cycle revisited: cycles and bifurcations. Math. Comput. Simul. 63, 505-527 (2003)

7. Sushko, I, Gardini, L, Puu, T: Regular and chaotic growth in a Hicksian floor/ceiling model. J. Econ. Behav. Organ. 75, 77-94 (2010)

8. Matsumoto, A, Szidarovszky, F: Continuous Hicksian trade cycle with consumption and investment time delays. J. Econ. Behav. Organ. 75, 95-114 (2010)

9. Hicks, JR: Methods of Dynamic Economic. Clarendon, Oxford (1985)

10. Kurihara, KK: Post-Keynesian Economics. Allen \& Unwin, London (1955)

11. Goodwin, RM: A growth cycle. In: Lundberg, E (ed.) The Business Cycle in Postwar World. Macmillan \& Co., London (1957)

12. Saura, D, Vázquez, FJ, Vegas, JM: Non-chaotic oscillations on some regularized Hicks models. J. Econ. Dyn. Control 22, 667-678 (1998)

13. Robinson, C: Dynamical Systems: Stability, Symbolic Dynamics and Chaos. Studies in Advanced Mathematics. CRC Press, Boca Raton (1995)

14. Lauwerier, HA: Two-dimensional iterative maps. In: Chaos. Nonlinear Sci. Theory Appl., pp. 58-95. Manchester University Press, Manchester (1986) 